\title{
Fractionating Polymer Microspheres as Highly Accurate Density Standards
}

\section{Citation}

Bloxham, William H., Jonathan W. Hennek, Ashok A. Kumar, and George M. Whitesides. 2015. "Fractionating Polymer Microspheres as Highly Accurate Density Standards." Anal. Chem. 87 (14) (July 21): 7485-7491. doi:10.1021/acs.analchem.5b01932.

\section{Published Version}

doi:10.1021/acs.analchem.5b01932

\section{Permanent link}

http://nrs.harvard.edu/urn-3:HUL.InstRepos:25040942

\section{Terms of Use}

This article was downloaded from Harvard University's DASH repository, and is made available under the terms and conditions applicable to Open Access Policy Articles, as set forth at http:// nrs.harvard.edu/urn-3:HUL.InstRepos:dash.current.terms-of-use\#OAP

\section{Share Your Story}

The Harvard community has made this article openly available.

Please share how this access benefits you. Submit a story.

\section{Accessibility}




\title{
Fractionating Polymer Microspheres as Highly Accurate Density
}

\section{Standards}

\author{
William H. Bloxham ${ }^{1,2}$, Jonathan W. Hennek ${ }^{1}$, Ashok A. Kumar ${ }^{1}$, \\ and George M. Whitesides ${ }^{1,3,4^{*}}$
}

${ }^{1}$ Department of Chemistry and Chemical Biology, Harvard University, 12 Oxford St., Cambridge, MA 02138

${ }^{2}$ Department of Physics, Harvard University, 17 Oxford St., Cambridge, MA, 02138

${ }^{3}$ Wyss Institute for Biologically Inspired Engineering, Harvard University, 60 Oxford St., Cambridge, MA 02138

${ }^{4}$ Kavli Institute for Bionano Science \& Technology, Harvard University, 29 Oxford St., Cambridge, MA 02138

"Corresponding author: gwhitesides@gmwgroup.harvard.edu 


\begin{abstract}
This paper describes a method of isolating small, highly accurate density-standard beads and characterizing their densities using accurate and experimentally traceable techniques. Density standards have a variety of applications, including the characterization of density gradients, which are used to separate objects in a variety of fields. Glass density-standard beads can be very accurate $\left( \pm 0.0002 \mathrm{~g} \mathrm{~cm}^{-3}\right)$, but are too large (3-7 $\mathrm{mm}$ in diameter) for many applications. When smaller density standards are needed, commercial polymer microspheres are often used. These microspheres have standard deviations in density ranging from 0.006 to $0.021 \mathrm{~g} \mathrm{~cm}^{-3}$; these distributions in density make these microspheres impractical for applications demanding small steps in density. In this paper, commercial microspheres are fractionated using aqueous multiphase systems (AMPS) - aqueous mixture of polymers and salts that spontaneously separate into phases having molecularly sharp steps in density — to isolate microspheres having much narrower distributions in density (standard deviations from 0.0003 to $0.0008 \mathrm{~g} \mathrm{~cm}^{-3}$ ) than the original microspheres. By reducing the heterogeneity in densities, this method reduces the uncertainty in the density of any specific bead and, therefore, improves the accuracy within the limits of the calibration standards used to characterize the distributions in density.
\end{abstract}

\title{
Introduction
}

Separation of small objects (e.g., cells, organelles) by density, and measurement of the densities of these objects, can be accomplished by flotation in fluid media having gradients in density. These gradients can be generated using a number of techniques, including Percoll gradients, ${ }^{1}$ sucrose gradients, ${ }^{2}$ magnetic levitation, ${ }^{3}$ ultracentrifugation, ${ }^{4}$ and density-gradient columns. ${ }^{5}$ Characterizing the density of an object as a function of position in the column or tube, however, is not straightforward, especially when high 
accuracy is required. The most reliable methods for calibrating density gradients use density standards - typically beads with densities measured by pycnometry or some other method. These measurements are, especially for standards purchased commercially, often not easy to trace, and the accuracy of the values stated is ultimately difficult to establish. Measurements of the densities of particles using flotation in density-gradient columns would be more straightforward if there were an experimentally traceable method of comparing densities to well-characterized density standards (e.g., small beads, with a low standard deviation in density, and with density known to a high accuracy).

In a number of fields, researchers exploit small differences in density between objects to enrich or separate them. White blood cells, for example, have several subtypes whose distributions in density overlap; when separating different components of blood, such as lymphocytes and monocytes, a difference in density of the separation fluid as small as $0.003 \mathrm{~g} \mathrm{~cm}^{-3}$ can reduce purity. ${ }^{6}$ In another example, single-walled carbon nanotubes with different electrical properties can be enriched using density differences of $0.002 \mathrm{~g} \mathrm{~cm}^{-3}$ induced by surfactant encapsulation. ${ }^{7}$ Table 1 provides a partial list of objects with small differences in density that have been separated. The use of internal standards is required to characterize the density gradient systems used to separate these objects. In many of these examples, small $(100-300 \mu \mathrm{m})$ density-standard beads are 
Table 1. A partial list of objects that have been separated using density-based methods in the literature, along with a typical difference in density between the separated samples.

\begin{tabular}{lllc} 
Object & $\begin{array}{l}\text { Approximate } \\
\text { Density } \\
\mathbf{( g ~ c m}\end{array}$ & $\begin{array}{l}\text { Typical Density } \\
\text { Difference } \\
\mathbf{( g ~ c m}\end{array}$ & Ref. \\
\hline X and Y Bovine Sperm & 1.10 & 0.0007 & 8 \\
Fragments of Different Glasses & $1.5-1.6$ & 0.002 & 9 \\
Surfactant-Encapsulated Carbon Nanotubes & 1.1 & 0.002 & 7 \\
White Blood Cell Types & 1.075 & 0.003 & 6 \\
Malaria Infected RBC from WBC & $1.053-1.086$ & 0.005 & 10 \\
Stem Cells from Bone Marrow & 1.084 & 0.006 & 11 \\
Forensic Evidence (e.g., Glitter) & $1.5-1.6$ & 0.01 & 9 \\
Amniotic Fluid Cell Types & $1.02-1.06$ & 0.01 & 12 \\
Real and Counterfeit Rulon Parts & $1.97-2.27$ & 0.02 & 13 \\
Sickled and non-Sickled Red Blood Cells & 1.1 & 0.02 & 14 \\
Organelles and Other Cellular Components & $1.12-1.20$ & 0.02 & 16 \\
Mixtures of Diesel and Kerosene Fuels & 0.83 & 0.02 & 17 \\
Recycled Plastics & 1.1 & 0.03 & 18 \\
Clay Particles & $1.8-2.8$ & 0.1 & \\
& & & \\
\hline
\end{tabular}

*Because the resolution of a density separation method can exceed its accuracy, the density differences between separated subsamples are often smaller than the accuracy to which the densities of those subsamples are determined. 
essential; large beads ( $>1 \mathrm{~mm})$ may be too large to fit physically within the system used for the measurement, or will spatially exclude other objects. The development of small density standards with a low standard deviation in density will improve the reliability of density-based separation and analysis techniques.

For densities between 0.8 and $1.6 \mathrm{~g} \mathrm{~cm}^{-3}$, the most accurate solid densitystandards are glass density-standard beads, which are commercially available in a variety of densities, and offer stated accuracies as small as $0.0001 \mathrm{~g} \mathrm{~cm}^{-3} \cdot{ }^{19,20}$ There are, however, limitations to the use of these beads: (i) they are expensive ( $\$ 90-140$ per bead), (ii) they are very delicate — a $20 \mathrm{~cm}$ drop will ruin a bead — and heavy usage may increase the chance of damage due to mishandling, (iii) few labs are equipped to verify that the density of a bead remains constant over time, (iv) their large size (4-7 $\mathrm{mm}$ in diameter) introduces uncertainties in their suspended heights (especially if gradients are not linear or the beads are asymmetric) and makes them too large for certain applications (e.g., as standards for MagLev or in density gradient columns), because they physically may not fit within a system, spatially exclude other objects, or cannot be localized-due to nonspherical shape - easily. We sought to create density standards that solved these problems.

This paper describes an experimentally simple method for isolating samples of highly accurate, small density-standard beads from samples of beads with large standard deviations in densities using aqueous multiphase systems (AMPS). AMPS are aqueous mixtures of polymers that spontaneously separate into two or more thermodynamically stable phases with different densities; ${ }^{21}$ these phases can be engineered to have steps in density at the interfaces as small as $0.0003 \mathrm{~g} \mathrm{~cm}^{-3}$. Commercial polymer microspheres (purchased from Cospheric LLC, diameters $\sim 250 \mu \mathrm{m})^{22}$ were fractionated in AMPS to extract specific sets of beads with narrower distributions in density. We characterize 
their distributions in density compared to standards having densities known to an accuracy of $\pm 0.0002 \mathrm{~g} \mathrm{~cm}^{-3}$ ("accuracy" meaning the closeness of a measurement to the true value, sometimes called "trueness"). We are able to achieve a standard deviation in density as small as $0.0003 \mathrm{~g} \mathrm{~cm}^{-3}$ - nearly two orders of magnitude better than similarly sized density-standard beads that are commercially available.

\section{Experimental Section}

Chemicals. Cesium bromide $(\mathrm{CsBr})$, manganese chloride tetrahydrate $\left(\mathrm{MnCl}_{2} \cdot 4 \mathrm{H}_{2} \mathrm{O}\right)$, Tween 20, and Ficoll (400K, BioXtra), were purchased from SigmaAldrich (St. Louis, MO, USA). Dextran (500K) was purchased from Spectrum

Chemicals (New Brunswick, NJ, USA). Liquid density standards were purchased from H \& D Fitzgerald Ltd. (Denbighshire, Wales, UK). All chemicals were used as received without purification.

Polymer Microspheres. Polymer microspheres were purchased from CoSpheric LLC (Santa Barbara, CA, USA). We tested the following beads: fluorescent violet density marker beads (DMB-FVIO-1.06, $\rho=1.062 \mathrm{~g} / \mathrm{ml}$ ), fluorescent red density marker beads (DMB-FRED-1.09, $\rho=1.092 \mathrm{~g} / \mathrm{ml}$ ), blue density marker beads (DMB-RBLU$1.08, \rho=1.084 \mathrm{~g} / \mathrm{ml})$, fluorescent blue density marker beads (DMB-FBLU-1.13, $\rho=$ $1.130 \mathrm{~g} / \mathrm{ml}$ ), blue microspheres (BLPMS-1.075, $\rho=1.077 \mathrm{~g} / \mathrm{ml}$ ), and red microspheres (BLPMS-1.12, $\rho=1.118 \mathrm{~g} / \mathrm{ml}$ ). Before fractionations in AMPS and microspheres were washed in a dilute $(\sim 0.01 \% \mathrm{v} / \mathrm{v})$ aqueous solution of Tween 20 to help prevent bubbles and reduce agglomeration.

Glass Density Standard Beads. Glass density standard beads were purchased from American Density Materials (Staunton, VA, USA) and are reported by the manufacturer to have uncertainties in density of $\pm 0.0002 \mathrm{~g} \mathrm{~cm}^{-3}$. 
Materials. Liquid density measurements were obtained using either a DMA-35 or a DMA-4500M U-tube densitometer from Anton-Paar. MagLev experiments were performed using a custom setup made of machined aluminum using $\mathrm{NdFeB}$ magnets from K\&J Magnets (Pipersville, PA, USA). AMPS solutions were centrifuged in $15 \mathrm{~mL}$ Falcon tubes (Corning Inc., Corning, NY, USA) using an Allegra 6R centrifuge (Beckman Coulter, Brea, California, USA).

Using AMPS to Fractionate Particles by Density. An AMPS with N-phases provides $\mathrm{N}+1$ interfaces at which objects may settle. For example, a 2-phase AMPS will have three interfaces: air - top phase, top phase - bottom phase, and bottom phase container. The sharp interfaces in AMPS offer a simple method to bin objects by density. ${ }^{14,21,23}$ AMPS offer several important benefits for the separation of objects by density: i) The volume and density of the phases can be tuned; ii) The difference in density between phases of the AMPS can be very small $\left(\geq 0.0003 \mathrm{~g} \mathrm{~cm}^{-3}\right)$; iii) AMPS concentrate objects at sharp interfaces, which allows for easy removal; iv) The interfacial tension in AMPS is very low $\left(100 \mathrm{~nJ} / \mathrm{m}^{2}-100 \mu \mathrm{J} / \mathrm{m}^{2}\right)^{24}$ because the principle component is water, and, therefore, the final position of objects as small as $200 \mu \mathrm{m}$ is determined primarily by density, and not by surface tension (see Supporting Information); v) Depending on composition, AMPS will generally phase-separate in less than three hours with centrifugation at $3200 \mathrm{~g}$, and even more quickly at higher speeds. We utilized these properties of AMPS to fractionate samples of beads with narrow distributions in density appropriate for use as density standards from collections of particles with wider distributions in density.

Use of Ficoll-Dextran-CsBr AMPS for Density Standard Isolation. In principle, any AMPS can be used to separate beads in order to reduce their distribution in density. We used two-phase AMPS composed of $\sim 10 \%(\mathrm{w} / \mathrm{v})$ Ficoll (a polysucrose, 
$\mathrm{MW} \sim 400 \mathrm{kDa}), \sim 6 \%(\mathrm{w} / \mathrm{v})$ dextran (a polyglucose, $\mathrm{MW} \sim 500 \mathrm{kDa}$ ), and different concentrations $(0-10 \%)$ of cesium bromide, $\mathrm{CsBr}$, to separate commercial standard beads into fractions with narrower distributions in density because this system gave the best balance of i) small steps in density $\left(\geq 0.0003 \mathrm{~g} \mathrm{~cm}^{-3}\right)$, ii) low surface tension between the two phases $\left(\sim 4 \mu \mathrm{J} / \mathrm{m}^{2}\right)$, iii) roughly equal volumes of phases for easy extraction, and iv) low viscosities of its components $\left(0.17 \mathrm{dL} \mathrm{g}^{-1}\right.$ for Ficoll ${ }^{21}$ and $0.50 \mathrm{dL} \mathrm{g}^{-1}$ for dextran $\left.{ }^{25}\right)$; solutions of polymers with higher viscosities are more difficult to manipulate. By varying the concentrations of Ficoll, dextran, and CsBr, the densities of the two phases, and other relevant parameters (phase volume ratio, separation time, viscosity), can be easily tuned (see Supporting Information).

\section{Commercial Microspheres Make Good Starting Materials. We used} commercial polymer microspheres (Cospheric LLC) with diameters of approximately $250 \mu \mathrm{m}$ as our starting materials. Polymer microspheres have five advantages as a starting material to produce density standards: (i) they are inexpensive (usually \$50-100 for several hundred thousand beads), (ii) they are available in a variety of colors, which allows different beads to be distinguished when used simultaneously, (iii) they are durable and easily handled when suspended in an aqueous solution containing surfactant, (iv) they are familiar to the research community and are widely used, and (v) their position, when suspended in a column, is easily determined .

Protocol for Creating Density Standards. Our method to isolate densitystandard beads with small distributions in density uses three steps (Figure 1): 1) A twophase AMPS with a small difference in density between its phases $(0.0003-0.0015 \mathrm{~g}$ $\mathrm{cm}^{-3}$ ) is mixed with commercial polymer microspheres with a large distribution in density. 2) The mixture is centrifuged to induce phase separation. As phase separation occurs, the beads collect at the interfaces based on their densities into three bins: a) beads 
that are less dense than the top phase of the AMPS $\left(\rho_{\text {bead }}<\rho_{\text {top }}\right)$, b) beads that are between the density of the top and bottom phases $\left(\rho_{\text {top }}<\rho_{\text {bead }}<\rho_{\text {bot }}\right)$, and c) beads that are denser than the bottom phase $\left(\rho_{\text {bot }}<\rho_{\text {bead }}\right)$. Only a very narrow range of densities $\left(\rho_{\text {top }}<\right.$ $\left.\rho_{\text {bead }}<\rho_{\text {bot }}\right)$ collect at the liquid-liquid interface, because the difference in density between the two phases is small. 3) Beads are collected from the liquid-liquid interface and washed to remove polymeric residues from the AMPS, and CsBr. A step-by-step protocol with images can be found in Figure S1 of the Supporting Information. We used a U-tube density meter (DMA 4500M, Anton Paar), described by the manufacturer as having an accuracy of $0.00005 \mathrm{~g} \mathrm{~cm}^{-3}$, to measure the densities of the phases of our AMPS. These data were used to define the range of densities of beads expected to collect at the interface. We then directly characterized the distribution in density of the beads using the linear gradient in density created by MagLev (see below), and examined the accuracy of the measurement by using glass density standard beads with known accuracies. Each AMPS is designed to collect beads of a particular density. After isolating and extracting beads, however, a small amount $(\sim 0.1-5 \% \mathrm{w} / \mathrm{v})$ of $\mathrm{CsBr}$ can be added to the AMPS in order to increase the density of both liquid phases.

After centrifugation, beads with a density between the new $\rho_{\text {top }}$ and $\rho_{\text {bot }}$ will collect at the liquid-liquid interface. This process can be repeated several times to isolate multiple density standards having different densities from the same original sample of beads (see Results and Discussion for more details).

\section{Magnetic Levitation Allows Easy Characterization of Distributions in}

Density. We used magnetic levitation (MagLev) ${ }^{3}$ to characterize the density and distribution in density of beads isolated using AMPS. In MagLev, an object is suspended (i.e., levitated) in a paramagnetic solution in a magnetic field (usually generated by two $\mathrm{NdFeB}$ permanent magnets oriented with like poles facing). ${ }^{3}$ 
Figure 1. Illustration of the process for producing density-standard beads from beads with wider distributions in density. 1) Beads are mixed with AMPS. 2) As the AMPS phase separates, buoyant forces cause the beads to collect at the interfaces based on density ( $\left.\rho_{\text {bead }}<\rho_{\text {top }}, \rho_{\text {top }}<\rho_{\text {bead }}<\rho_{\text {bot }}, \rho_{\text {bot }}<\rho_{\text {bead }}\right) 3$ ) Beads are extracted from the interface containing densities $\rho_{\text {top }}<\rho_{\text {bead }}<\rho_{\text {bot }}$ and washed to remove polymers. Aliquots of the phases above and below the interface are collected and their densities measured in order to determine the densities of the beads collected at the interfaces. See Supporting Information Figure S1 for images of a step-by-step protocol.

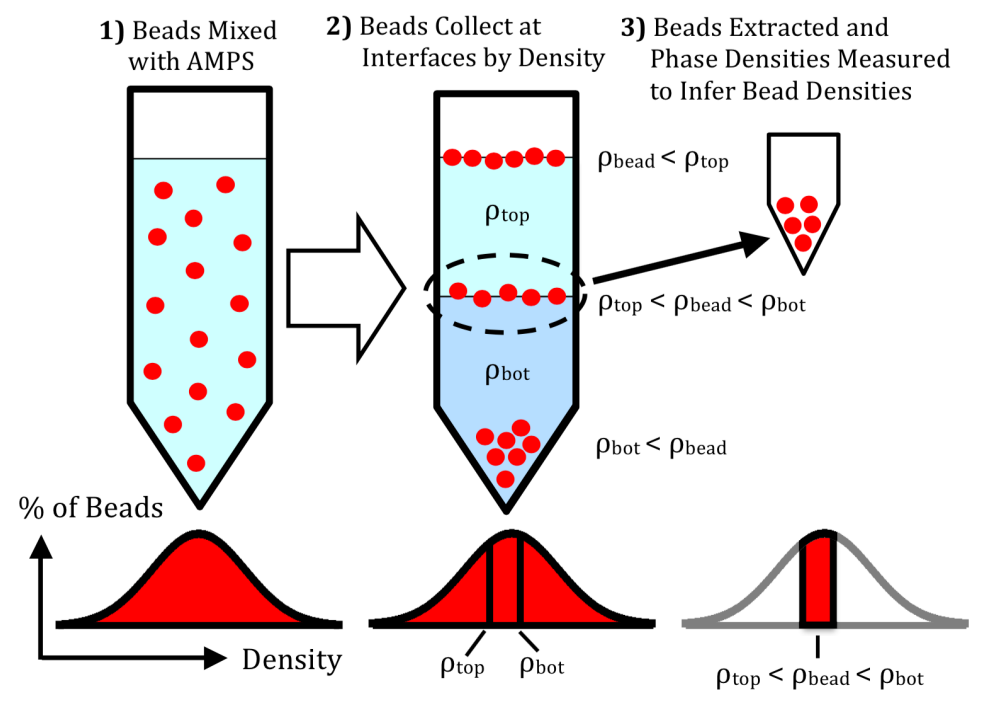


MagLev can make density measurements with an accuracy of $0.0002 \mathrm{~g} \mathrm{~cm}^{-3} .^{3}$ In the configuration of MagLev used here, the gravitational and magnetic forces balance in a way that generates an approximately linear relationship between levitation height and the density of an object. ${ }^{3}$ MagLev can simultaneously enable the measurement of the densities of multiple particles in a sample, provided the particles are small enough that they do not spatially exclude each other from levitating at the heights dictated by their densities. MagLev is, therefore, a useful tool for characterizing the distributions in density of small particles. Determining the absolute density, rather than the distribution in densities, requires standards - we discuss that process in the Results and Discussion and in detail in the Supporting Information.

We used MagLev first to characterize the distributions in density of the microspheres as purchased. Then, after fractionating the microspheres with our AMPS, we once again characterized their distributions in density in order to determine quantitatively to what extent our fractionations narrowed the distributions in density (using standard deviation as the quantification of a distribution in density). See Supporting Information for descriptions of the MagLev setup, and the details of the calculation.

\section{Results and Discussion}

Analysis of Commercial Density Standard Microspheres. We used MagLev, as described above, to analyze the distributions in density of commercially available polymer microspheres (CoSpheric LLC, $\mathrm{N}=78-191$ beads). The manufacturer stated a "tolerance in the mean density of the beads" of $\pm 0.005 \mathrm{~g} \mathrm{~cm}^{-3}$ for the microspheres labeled as density marker beads. ${ }^{22,26}$

We found the commercial microspheres had standard deviations in density from $0.006 \mathrm{~g} \mathrm{~cm}^{-3}$ to $0.021 \mathrm{~g} \mathrm{~cm}^{-3}$ with an average standard deviation in density of $0.011 \mathrm{~g} \mathrm{~cm}^{-}$ 
3. In most cases the stated density lay outside the $25^{\text {th }}$ or $75^{\text {th }}$ percentile. Figure 2 shows the distribution in density for each of the commercial microspheres we characterized. Using AMPS to Isolate Density Standards. To demonstrate the method, we developed a series of AMPS to isolate beads with tight distributions in density and performed a series of isolations. For each isolation, we started with a sample of commercial densitystandard beads and narrowed the wide distribution in density of those beads via AMPS filtration (i.e. fractionation followed by isolation of the beads). We designed the AMPS to produce beads whose densities were approximately the stated densities of the original beads. AMPS can also be designed to isolate beads that are outliers in density rather than to isolate those that are in the center of the distribution. Isolating outliers would, however, reduce the yield of beads with a narrow distribution in density (we demonstrate this approach later in the Results section). See Supporting Information for details of the composition of the AMPS used in each isolation.

After fractionation and extraction, the isolated beads were characterized using MagLev. Their distributions in density were compared to the distributions in densities of the original beads. Figure 3 shows a significant decrease in the width of the distributions in density of the beads. The standard deviations in density of the isolated beads ranged from $0.0003 \mathrm{~g} \mathrm{~cm}^{-3}$ to $0.0008 \mathrm{~g} \mathrm{~cm}^{-3}$ - that is, a reduction by a factor of $18-39$ relative to the original sample of beads. The yield of isolation was approximated by measuring the mass of beads before AMPS fractionation, calculating the number of beads from this quantity and the reported size and density, and comparing this to the number of beads after filtration. These yields were approximately $0.1 \%$ to $0.2 \%$. As our systems were designed to reject the majority of the beads, a low yield was expected; the protocol we describe is non-destructive and, thus, rejected beads can be recovered and reused. 
Figure 2. Box plots showing the distribution in density for 200-300 $\mu \mathrm{m}$ diameter polymer microsphere purchased from CoSpheric LLC. In these box plots and all others in this paper, the box extends from the $25^{\text {th }}$ percentile to the $75^{\text {th }}$ percentile and with the central line marking the median density. The whiskers extend from the end of the box a maximum of 1.5 times the change in density from the $25^{\text {th }}$ to $75^{\text {th }}$ percentile; whiskers do not extend beyond the most extremal datum. Additional points represent outliers. The colors of the boxes represent the color of the beads as purchased. Mean densities and distributions in density were measured using MagLev $(n=78-191)$. The dotted line represents a 1:1 relationship between the density stated by the manufacturer and the density as measured in our experiments.

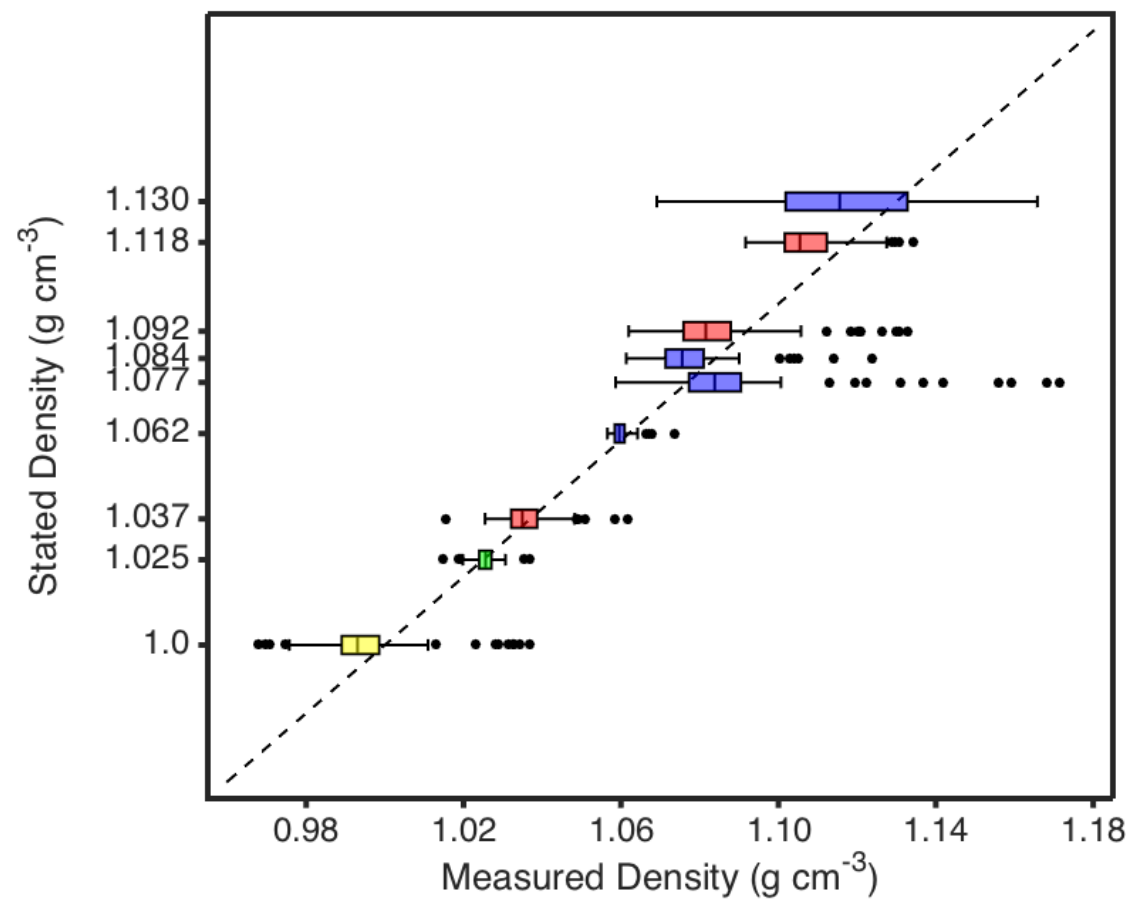


Figure 3. Box plot showing polymer bead distributions in density before $(n=78-191)$ and after $(\mathrm{n} \sim 10)$ filtration by an AMPS. The dashed line indicates the stated densities of the original beads. Isolation in AMPS produces beads that have a mean density closer to the stated density and narrower distributions in density. The colors of the boxes represent the color of the beads as purchased.
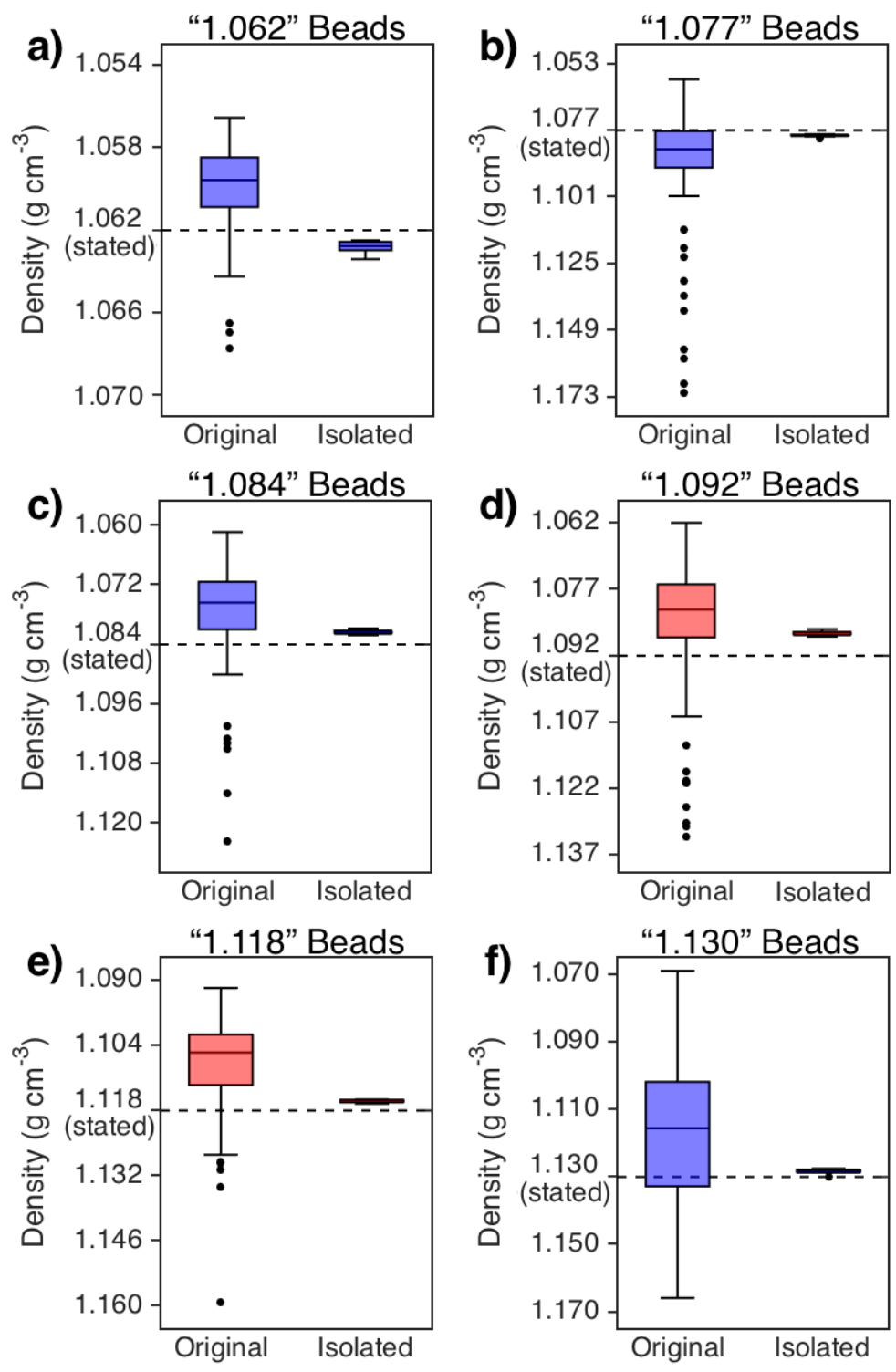
There may also be uses for sets of beads in which a selected, narrow density range is missing.

Isolating Multiple Sets of Standards from the Same Original Sample. To demonstrate the ability of our systems to isolate multiple sets of density standards from the same original sample of beads, we designed a system capable of performing a series of fractionations. We started by mixing commercial density-standard microspheres with a stated density of $1.130 \mathrm{~g} \mathrm{~cm}^{-3}$ (CoSpheric LLC) with an AMPS composed of $10.2 \%$ (wt/vol) Ficoll, 6.36\% (wt/vol) dextran, and 5.58\% (wt/vol) CsBr. Centrifugation at 3200 $g$ induced phase separation of the system and we extracted beads from the liquid-liquid interface. We added $0.05 \%$ (wt/vol) Ficoll, $0.03 \%$ (wt/vol) dextran, and $1.25 \%$ (wt/vol) $\mathrm{CsBr}$ to the system in order to increase the density of both phases by approximately 0.05 $\mathrm{g} \mathrm{cm}^{-3}$. The addition of Ficoll and dextran was necessary to compensate for the narrowing of the difference in density between the two phases resulting from $\mathrm{CsBr}$ addition; $\mathrm{CsBr}$ enters the less dense dextran-rich phase slightly more readily than it enters the Ficoll-rich phase. (See Supporting Information for more detail on tuning a Ficolldextran-CsBr AMPS.) We centrifuged the altered system and again extracted beads from the liquid-liquid interface. Repeating this process, we isolated a total of five sets of beads.

MagLev enabled characterization of the density and distribution in density of each sample of beads. As shown in Figure 4, we successful isolated density-standard beads with narrow distributions in density at five different densities from the same original sample of beads. In principle, this strategy could be repeated many times to extract nearly all of the beads into a series of fractions each with narrow distributions in density. 
Figure 4. Boxplots showing the distributions in density for extracted beads after a series of fractionations using an AMPS in which the $\mathrm{CsBr}$ and polymer concentrations were increased between each fractionation. The distribution in density of the original sample $(\mathrm{n} \sim 100)$ is shown as a comparison. Each fractionation produced approximately 8-10 beads. Inset shows the density of each bead from Fraction 1.

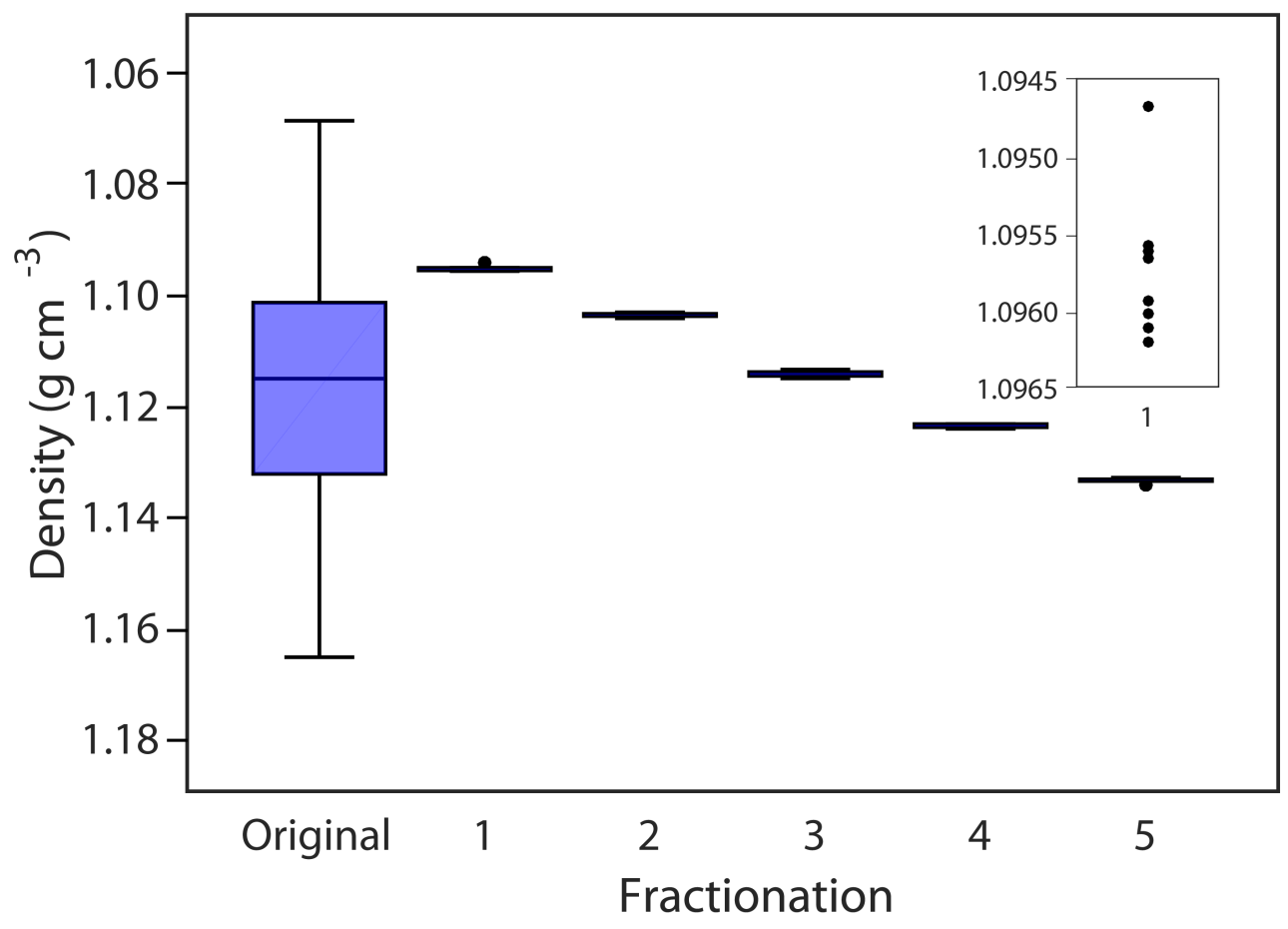




\section{Accurate Measurement of the Densities of the Phases of AMPS Predicts}

Isolated Bead Densities. The analytical technique we call "MagLev" is not common in most laboratories. We, therefore, sought to confirm that the densities of the beads fractionated by AMPS could be inferred by measuring the phases of the AMPS from which they were separated using a U-tube densitometer (a more common piece of equipment), in place of measuring the densities of the beads by levitating them in MagLev. Figure 5 compares the distributions in density of six different sets of beads as measured by MagLev (colored circles) to the densities of the top and bottom phases (black lines) from which the beads were extracted. We measured the densities of each phase three times with a DMA 4500M (Anton Paar). The range of measured densities for each phase is given as a grey band in Figure 5; the black line in the mean value. In each MagLev measurement, we used three or more glass density standards, having an accuracy of $0.0002 \mathrm{~g} \mathrm{~cm}^{-3}$, as internal reference standards for the measurement.

We found the distributions in density of the beads from each isolation to be slightly offset from the densities of the two phases: in each case the distribution in density of the isolated beads extended beyond the density of one phase while not reaching the other. This offset was generally $\sim 0.001 \mathrm{~g} \mathrm{~cm}^{-3}$. The total width of each distribution in density, however, was roughly equal to the difference in density between the two phases. This observation suggests that surface tension did not tend to hold beads at the interface when their densities were not between the densities of the two phases; we would have expected to see distributions in densities wider than the difference in density between the two phases if surface tension played a major role. Instead, errors between Utube and MagLev measurements may explain the discrepancies. 
Figure 5. Comparison of MagLev and U-tube density measurements. Beads were isolated using AMPS, the densities of whose phase were measured by U-tube densitometry. The intended density of the beads is labeled above each inset (e.g. " 1.062 beads"). The densities of the isolated beads were measured with MagLev and compared to the densities of the phases of the AMPS used to isolate each bead. Grey regions show the range of values for repeated U-tube density measurements of top and bottom phases; black lines show mean values. Colored dots show densities of isolated beads as measured by MagLev. The x-axis is unitless and exists to provide separation of the data points.

\section{a) 1.062 Beads}

b) 1.077 Beads
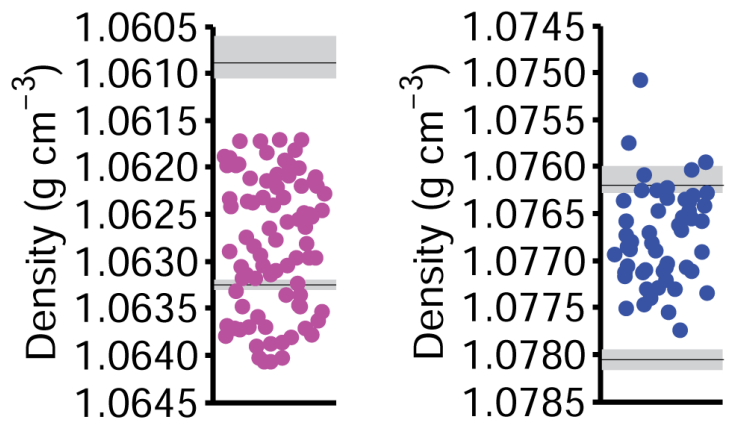

\section{c) 1.084 Beads}

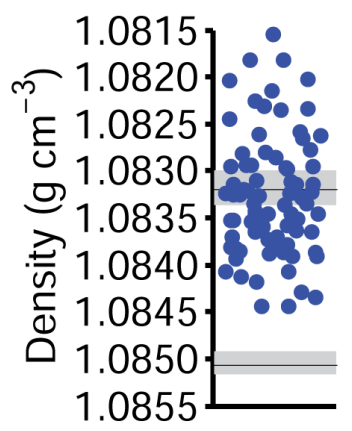

\section{d) 1.092 Beads}

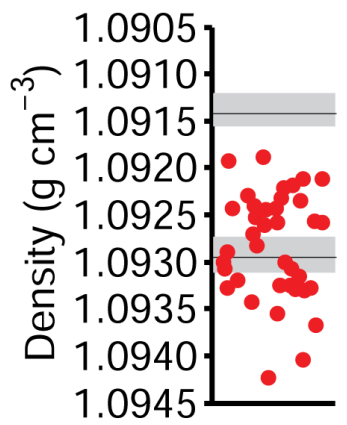

\section{e) 1.118 Beads}

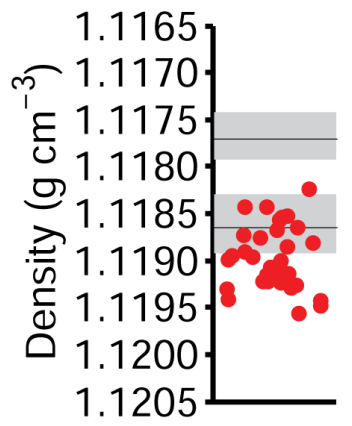

\section{f) 1.130 Beads}

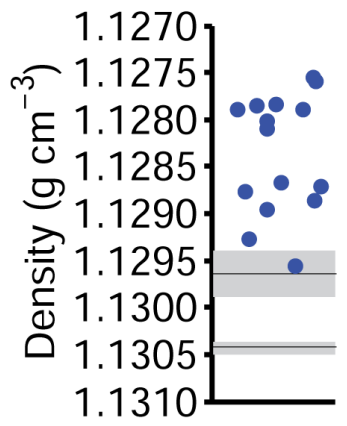


Several sources of error could contribute to the differences between the MagLev and U-tube measurements, including (i) aeration or evaporation of the phase samples between collection and measurement, (ii) systematic errors in measurements from the DMA 4500M Density Meter, related to both the instrument and technique of the operator (measuring liquid density standards [H\&D Fitzgerald] having densities $0.998202 \mathrm{~g} \mathrm{~cm}^{-3}$ [distilled water], $1.037215 \mathrm{~g} \mathrm{~cm}^{-3}\left[10 \% \mathrm{w} / \mathrm{v}\right.$ dextrose in water], and $1.249076 \mathrm{~g} \mathrm{~cm}^{-3}$ $\left[27 \% \mathrm{w} / \mathrm{v}\right.$ sodium bromide in water] revealed inaccuracies of $0.00008-0.00028 \mathrm{~g} \mathrm{~cm}^{-3}$, albeit for only one measurement per standard; measuring a series of dextran and Ficoll solutions [Table S1] revealed a standard deviation of $0.00024-0.00059 \mathrm{~g} \mathrm{~cm}^{-3}$ across 10 measurements of solutions of relevant concentrations of Ficoll and dextran), (iii) nonlinearities in the magnetic field of the MagLev device, ${ }^{3}$ (iv) inaccuracies in the densities of the glass density-standard beads (the manufacturer stated accuracy to 0.0002 $\mathrm{g} \mathrm{cm}^{-3}$; neutral buoyancy experiments using the DMA 4500M Density Meter to measure the densities of the neutral buoyancy solutions revealed inaccuracies of 0.00009 to $0.00060 \mathrm{~g} \mathrm{~cm}^{-3}$ relative to the DMA $4500 \mathrm{M}$ ), and (v) inaccuracies in determining the heights of the (not perfectly spherical) glass beads in the MagLev device-a $0.1 \mathrm{~mm}$ uncertainty in height is equivalent to a $0.0002 \mathrm{~g} \mathrm{~cm}^{-3}$ uncertainty in density for the concentration of paramagnetic salt used here $\left(\sim 0.3 \mathrm{M} \mathrm{MnCl}_{2}\right)$.

To quantify the improvement in the accuracy to which the densities of the microspheres are characterized, we calculated the root mean squared (RMS) difference between the densities of the beads as measured by MagLev and the expected density of the beads - for the beads isolated by AMPS, we define the expected density of the beads as the average of the densities of the two phases of the AMPS from which they were extracted - the best guess at the density of the isolated beads one could make solely from U-tube data. For the as-purchased commercial microspheres, we define the expected 
density of the beads as the density reported by the manufacturer. These values are shown

in Table 2. The commercial microspheres had RMS deviations from their stated densities of $0.0032 \mathrm{~g} \mathrm{~cm}^{-3}$ to $0.0239 \mathrm{~g} \mathrm{~cm}^{-3}$. The microspheres isolated by AMPS had RMS deviations from their expected densities of $0.0006 \mathrm{~g} \mathrm{~cm}^{-3}$ to $0.0016 \mathrm{~g} \mathrm{~cm}^{-3}$, a 3 to 34-fold decrease. These values are not the total accuracy of each measurement because they do not capture the uncertainty of MagLev measurements (e.g. the $0.0002 \mathrm{~g} \mathrm{~cm}^{-3}$ uncertainty of glass density standard beads used as references); nevertheless the improvement in the RMS deviations reduces a potentially large source of uncertainty that contributes to the total accuracy.

The U-tube inferences agreed with the MagLev measurements to an accuracy of roughly $0.0010 \mathrm{~g} \mathrm{~cm}^{-3}$. Considering the strong relationship between the width of the distributions in density of the isolated bead and the differences in density between the phases, we believe that beads are settling at interfaces based almost entirely based on density (not surface tension), and that the discrepancies are the result of relative errors between U-tube and MagLev density measurements. (The discrepancies are on the same order of magnitude as many of potential sources of error in the two measurements listed above.) Without any strong reason to believe MagLev measurements to be more accurate than U-tube measurements, inferring beads densities from U-tube measurements will likely be a better choice than performing MagLev measurements, as the inferences from U-tube measurements (i) are experimentally simpler, (ii) require equipment a lab working with density is likely already to have, and (iii) will result in isolated beads with densities that will be traceable to the same U-tube device likely used for other density measurements in the lab. 
Table 2. Expected densities and root mean squared (RMS) deviations from the expected densities for commercial microspheres and microspheres isolated by AMPS.

\begin{tabular}{cccc}
\hline \multicolumn{2}{c}{ Commercial Microspheres } & \multicolumn{2}{c}{ Microspheres Isolated by AMPS } \\
$\begin{array}{c}\text { Expected Density } \\
\left(\mathrm{g} \mathrm{cm}^{-3}\right)^{[\mathrm{a}]}\end{array}$ & $\begin{array}{c}\text { RMS Deviation From } \\
\text { Expected Density }\left(\mathrm{g} \mathrm{cm}^{-3}\right)\end{array}$ & $\begin{array}{c}\text { Expected Density } \\
\left(\mathrm{g} \mathrm{cm}^{-3}\right)^{[\mathrm{b}]}\end{array}$ & $\begin{array}{c}\text { RMS Deviation From } \\
\text { Expected Density }\left(\mathrm{g} \mathrm{cm}^{-3}\right)\end{array}$ \\
\hline 1.062 & 0.0032 & 1.0621 & 0.0010 \\
1.077 & 0.0212 & 1.0771 & 0.0006 \\
1.084 & 0.0118 & 1.0841 & 0.0011 \\
1.092 & 0.0153 & 1.0922 & 0.0009 \\
1.118 & 0.0135 & 1.1182 & 0.0011 \\
1.130 & 0.0239 & 1.1300 & 0.0016 \\
\hline
\end{tabular}

[a] stated by manufacturer

[b] estimated as the average of the density of the top and bottom phase of the AMPS used for fractionation 


\section{Conclusions}

We have demonstrated an experimentally traceable method of isolating small density-standards with high accuracy and small distributions in density. AMPS isolate commercially available beads at sharp interfaces between liquid layers, and provide a standard deviation in density 18 to 39 times smaller than commercial density standards of the same size. We show that U-tube densitometry can be used to estimate the density of the beads and reduce a major source of uncertainty in density (i.e., heterogeneity in density) by more than an order of magnitude. The protocol described in this work, therefore, requires only a centrifuge and a U-tube densitometer to produce density standards with small distributions in density and densities known accurately. AMPS are also easily scalable; thousands of beads can be isolated in a single fractionation.

Many density-based applications require small density standards for calibration. Small $(100-300 \mu \mathrm{m})$ commercially available density standards are, however, less accurate than large (>4 mm) density standards by roughly two orders of magnitude, due to the difficulties of accurately measuring the volume of small objects. Reproducibility and traceability in density measurements are important to ensure accurate reporting of (and reproducibility of) experimental methods. A U-tube densitometer, which can be easily calibrated and verified in-house, is a simple method to determine the density of AMPS accurately, and to infer the density of beads fractionated by an AMPS.

There are two alternatives to the density standards described in this paper: (1) glass density-standard beads and (2) commercial polymer microspheres (i.e., the unfractionated beads used in this study). The use of glass density-standard beads may be warranted when (i) only large-volume (>20 mL) experiments are performed, (ii) experiments do not require high spatial resolution (where a large bead might exclude the object of interest), or (iii) cost is not a consideration. Commercial polymer microspheres 
may be most useful in cases when the uncertainty in density does not need to be less than $0.05 \mathrm{~g} \mathrm{~cm}^{-3}$, or where the cost of the beads fractionated using AMPS is too high.

The standards described in this paper would be best for experiments (i) performed on small volumes and/or with small objects, (ii) that require high degrees of accuracy, and traceability, (iii) involving objects with small differences in density or (iv) that may change in requirements over time-for example, in a research lab where the target density may change depending on the project and, thus, the cost and time of purchasing glass density standards becomes a burden. The process of fractionating commercial beads using AMPS described here enables the isolation of traceable, accurate beads having a broad range of densities. These beads have a smaller distribution in density than any small $(<1 \mathrm{~mm})$ density standards presently available and may, therefore, expand the applications for which density-standard microspheres can be used. The ability to obtain highly accurate small density-standard beads with a relatively simple methodcentrifugation in AMPS — should improve standardization and rigor in density-based analyses.

\section{Acknowledgements}

The authors thank Charles Mace for helpful discussions and assistance with spinning drop measurements, and Dennis Meyers at Anton Paar for providing use of a DMA 4500M. This work was supported by an NIH-SBIR grant 1R43HL 123818-01 and by

CAMTech award \#221501 from MGH. A.A.K. acknowledges financial support from the Office of Naval Research through a National Defense Science and Engineering Fellowship. W.H.B. was supported by the Harvard College Program for Research in Science and Engineering (PRISE) and the Harvard College Research Program (HCRP). 


\section{References}

(1) GE-Healthcare. Cell Separation Media - Methodology and Applications; 2007.

(2) Arrowood, M.; Sterling, C. J. Parasitol. 1987, 73, 314-319.

(3) Mirica, K. A.; Shevkoplyas, S. S.; Phillips, S. T.; Gupta, M.; Whitesides, G. M. J. Am. Chem. Soc. 2009, 131, 10049-10058.

(4) Cole, J. L.; Lary, J. W.; P Moody, T.; Laue, T. M. Methods Cell Biol. 2008, 84, 143-179.

(5) Lindsley, C. H. J. Polym. Sci. 1960, 46, 543-545.

(6) Boyum, A.; Fjerdingstad, H. B.; Martinsen, I.; Lea, T.; Lovhaug, D. Scand. J. Immunol. 2002, 56, 76-84.

(7) Arnold, M. S.; Green, A. A.; Hulvat, J. F.; Stupp, S. I.; Hersam, M. C. Nat. Nanotechnol. 2006, 1, 60-65.

(8) Gledhill, B. L. Gamete Res. 1988, 20, 377-395.

(9) Lockett, M. R.; Mirica, K. A.; Mace, C. R.; Blackledge, R. D.; Whitesides, G. M. J. Forensic Sci. 2013, 58, 40-45.

(10) Trang, D. T. X.; Huy, N. T.; Kariu, T.; Tajima, K.; Kamei, K. Malar. J. 2004, 3 , 7.

(11) Juopperi, T. A.; Schuler, W.; Yuan, X.; Collector, M. I.; Dang, C. V; Sharkis, S. J. Exp. Hematol. 2007, 35, 335-341.

(12) Cousineau, J.; Potier, M.; Dallaire, L.; Melançon, S. B. Prenat. Diagn. 1982, 2, 241-249.

(13) Hennek, J. W.; Nemiroski, A.; Subramaniam, A. B.; Bwambok, D. K.; Yang, D.; Harburg, D. V; Tricard, S.; Ellerbee, A. K.; Whitesides, G. M. Adv. Mater. 2015, 27, 1587-1592.

(14) Kumar, A. A.; Patton, M. R.; Hennek, J. W.; Lee, S. Y. R.; D’Alesio-Spina, G.; Yang, X.; Kanter, J.; Shevkoplyas, S. S.; Brugnara, C.; Whitesides, G. M. Proc. Natl. Acad. Sci. U. S. A. 2014, 111, 14864-14869.

(15) Lodish, H.; Berk, A.; Zipursky, S. L.; Matsudaira, P.; Baltimore, D.; Darnell, J. Purification of Cells and Their Parts; W. H. Freeman, 2000.

(16) Yadav, S. R.; Murthy, V. K.; Mishra, D.; Baral, B. Int. J. Environ. Sci. Technol. 2013, 1, 253-255.

(17) Richard, G. M.; Mario, M.; Javier, T.; Susana, T. Resour. Conserv. Recycl. 2011, $55,472-482$.

(18) Halma, G. Clay Miner. 1969, 8, 59-69.

(19) H\&D Fitzgerald. Gradient Column Floats. Available at density.co.uk/products/gradient-column-floats/. Accessed March 20, 2015.

(20) American Density Materials. Accurate Density Floats. Available at www.americandensitymaterials.com. Accessed March 11, 2015.

(21) Mace, C. R.; Akbulut, O.; Kumar, A. A.; Shapiro, N. D.; Derda, R.; Patton, M. R.; Whitesides, G. M. J. Am. Chem. Soc. 2012, 134, 9094-9097.

(22) Cospheric-LLC. Density Marker Bead Technical Data Sheet.

(23) Kumar, A. A.; Lim, C.; Moreno, Y.; Mace, C. R.; Syed, A.; Van Tyne, D.; Wirth, D. F.; Duraisingh, M. T.; Whitesides, G. M. Am. J. Hematol. 2014, 90, 31-36.

(24) Hatti-Kaul, R. Mol. Biotechnol. 2001, 19, 269-277.

(25) Masuelli, M. A. J. Polym. Biopolym. Phys. Chem. 2013, 1, 13-21.

(26) Hennek, J. W. Personal Communication. 


\section{Table of Contents Graphic}

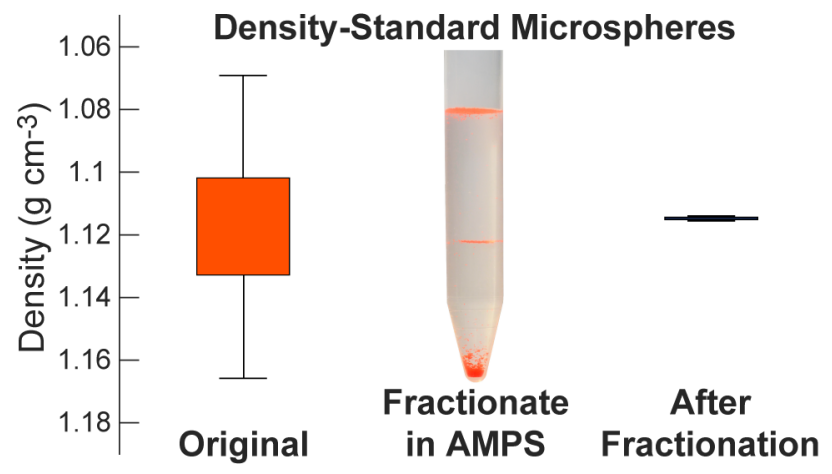

